\title{
SECURITY SYSTEM WITH FACE RECOGNITION, SMS ALERT AND EMBEDDED NETWORK VIDEO MONITORING TERMINAL
}

\author{
J. Shankar Kartik ${ }^{1}$, K. Ram Kumar ${ }^{2}$ and V.S. Srimadhavan ${ }^{3}$ \\ ${ }^{123}$ Department of Electronics and Communication Engineering, SRM Easwari \\ Engineering College, Anna University
}

\begin{abstract}
Even though there are various security systems consuming large power are available in market nowadays, robbery rate is very high. We are proposing a novel system to prevent robbery in highly secure areas with lesser power consumption. This system has face-recognition technology which grants access to only authorized people to enter that area. If others enter the place without access using some other means, then the system alerts the security personnel and streams the video captured by the security camera. The facerecognition is done using PCA algorithm. The video transmitted is compressed and transmitted by ENVMT. By using this ENVMT, the video can play with lesser bandwidth consumption, latency and jitter.
\end{abstract}

\section{KEYWORDS}

ENVMT, MPEG-4, PCA analysis, ISS, ESS

\section{INTRODUCTION}

Robbery has become common in our day to day life. Countering it, security systems with CCD cameras are commercially available. These systems are powered the entire time and they capture video throughout the day, hence consuming large amount of electricity. In most of the places, remote surveillance is needed. These systems, capture video, transmit it through the internet to the server, and from the server it is transmitted to the client. For this, large amount of bandwidth is consumed and there available for longer time. Even in tight security areas, these drawbacks are present. We propose a novel is some latency and jitter in transmitting the video. Sometimes, if the network is busy, the video is not smart security system, which can transmit video with lesser bandwidth consumption, latency and jitter.

For any smart security system, the two elements that ensure perfect security are the Video monitoring system and the access granting system. The proposed system consists of ENVMT technology for video monitoring and Face-recognition for granting access

\section{ARCHITECTURE OF THE SYSTEM}

The ISS is triggered by the micro-controller 2 which is controlled by micro-controller 1 in a master slave fashion. We can have any number of This section introduces the overall architecture of the proposed system and describes the components in detail.

DOI : $10.5121 /$ ijsptm.2013.2502 
The system architecture is shown in figure 1. The overall system consists of two sub-systems. They are the Internal Sub-system (ISS) and the External Sub-system (ESS). The ISS consists of the ENVMT, a GSM module and a control module for controlling the ISS. The ESS consists of the Face-recognition module and a control module for controlling the ESS and for triggering the ISS.

In ESS, the face recognition is done using MATLAB and the control is done by using two 8-bit micro-controllers with one serially communicating with the face-recognition module and other for triggering the ISS and its operations. There is an single primary camera which captures the image of the person who is waiting by the door for access and sends it to the face-recognition module for computation. In order to save power an external sensor is used for sensing movement outside the secure area and if any movement is present just near the door, say a person walking in just before the door for access, the external sensor senses motion and triggers the microcontroller 1 , which inturn triggers the face recognition module. Hence the Primary camera is just turned on when it needs to take the picture and it is turned off otherwise internal sensors for sensing motion inside the room. We can use each sensor for triggering each ENVMT or all the internal sensors for triggering a single ENVMT through the micro-controller 2. Both the External and Internal sensors are motion sensors, where we can use PIR sensor modules (Passive Infra-red Sensors) preferably.

The ENVMT captures video signals through the secondary camera, transforms analog signals to digital signals, compresses the ${ }^{\text {signals }}$ and releases to the network. The ENVMT is implemented using ARM processor. The GSM module is used to sending SMS alert to the client or the registered numbers stored in the EEPROM of the micro-controller.

The ENVMT captures video signals through the secondary camera, transforms analog signals to digital signals, compresses the ${ }^{\text {signals }}$ and releases to the network. The ENVMT is implemented using ARM processor. The GSM module is used to sending SMS alert to the client or the registered numbers stored in the EEPROM of the micro-controller.

\section{HaRdware Design Of The SyStem}

This section includes the hardware design of the proposed system and describes each component in detail.

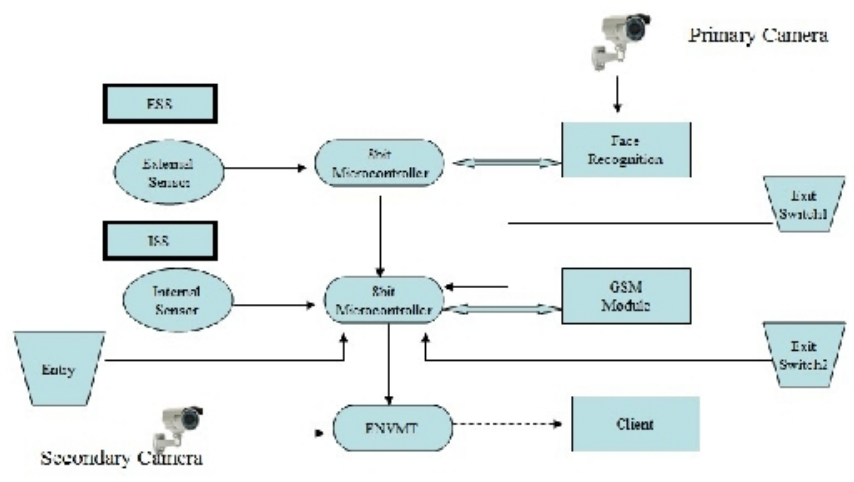

Figure 1 - Architecture of the system 


\subsection{ESS ARCHITECTURE:}

The ESS shown in the architecture consists of 4 hardware components. They are the External Sensor, The 8-bit micro-controller 1, the Face-recognition module and the Primary camera.

External Sensor: The external sensor is a simple motion sensor which is used to detect presence of people in front of the door waiting for access. Here we use a simple PIR sensor module, which consists of a PIR sensor and a simple circuit for making it send digital signals which is easier and faster to interface with a micro-controller. Hence, the module gives an output HIGH if there is presence of motion, i.e., any person in front of the door and an output LOW if there is no motion. The HIGH is used for triggering the rest of the module.

8bit Microcontroller 1:This controller is used for reading the output signal from the External Sensor, for serial communication with the face-recognition module and for triggering the 8 bit Microcontroller 2. Both the controllers used are of AVR architecture.It doesn't take any action other than reading the output of the External sensor until a HIGH is obtained at the pin reading the sensor. When it reads a HIGH it triggers the Face-recognition module and waits for the response of the Face-recognition module whether to grant access or not and depending upon the response, necessary action is taken in triggering the micro-controller 2.

Face-recognition Module: The Face-recognition module is based on PCA (Principal Component Analysis) based on Eigen-faces and it is programmed using MATLAB/Open CV. It is executed in a PC/FPGA which can be chosen depending on the need or the environment where the system is implemented. It is programmed to serially communicate with the micro-controller 1 and capture an image using the Primary camera and process the image and give a response to the microcontroller 1 whether the person is recognized or not.

Primary Camera: It is a small camera kept in a suitable position to take the image of the person facing it and send it to the Face-recognition module. It is controlled by the Face-recognition module.

\subsection{ISS ARCHITECTURE}

The ISS shown in the architecture consists of 5 hardware components. They are the internal sensor, 8bit microcontroller 2, GSM modem, the entry button switch, 2 exit button switches and the ENVMT with a secondary camera.

Internal sensor: It is a motion sensor, which is the same type as the external sensor. It is used for detecting motion inside the room and sends signals to the micro-controller 2.

8bit Microcontroller 2: It controls the ISS depending upon the signals received from the microcontroller 1.It can turn on the ISS or turn it off. It is serially connected to a GSM modem through its UART ports and hence can send a SMS to the registered phone numbers. It receives signals from the internal sensor and if it is a HIGH, it triggers the ENVMT and makes the GSM modem to send SMS alerts for the registered phone numbers. It also receives signals from the entry and exit switches and takes the necessary actions.

GSM modem: It consists of a SIM 300 moduleand additional circuitry for power management. It is serially connected to the micro-controller 2 and hence can receive the AT (Attention commands) from the controller and respond to it respectively.

Entry switch: It is used to indicate the system that the person has entered the room and hence it can close the access door and hence keep the ISS turned off. 
Exit switch 1: It is used to indicate the system that person is about the leave the room and hence it needs to open the door for them to exit.

Exit switch 2: It is used to indicate the system that the person has left and hence it can close the access door and restart the system's usual functionality.

ENVMT: It is the abbreviation of embedded network video monitoring terminal. It is implemented using an ARM processor. The hardware system includes processor, video-capture devices, and user's mobile phone or a pc to receive video information. In this study, ARM920Tbased S3C2440 processor is chosen to complete the core control; OV9650CMOS camera is used as a video-capture device which is our secondary camera; and the user's phone/pc is connected to the Internet to receive video information to achieve real-time monitoring. The hardware design block diagram of the ENVMT is shown in Figure 2.

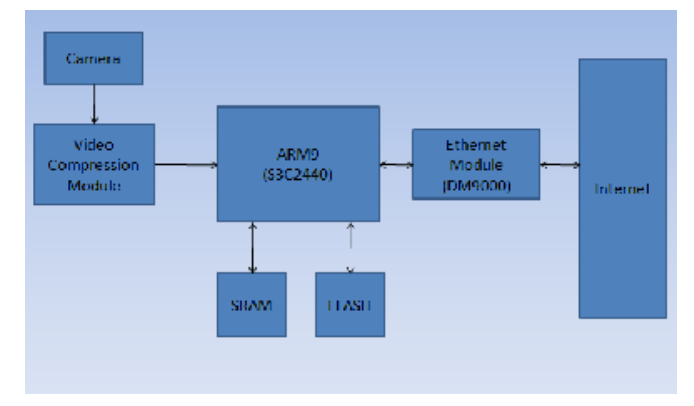

Figure 2 Block Diagram of ENVMT using ARM

S3C2440 processor based on ARM920T architecture is selected in the system; its expansion board provides the IO resources associated with our design (Huang et al, 2010). Ethernet interface provides chip CS8900A with $10 \mathrm{M}$ Ethernet, chooses integrated isolation transformer RJ4 interface, and with the ACT, LINK indicator.

In this design, it has dual role: used as the port for downloading the WinCE operating system in the design progress, and the carrier of the transmission channel after the system design. Digital camera circuits provides the standard digital camera interfaces, used to link a variety of camera modulesexternally.OV9650 CMOS camera with 1.3 million pixels is selected in the built system. Particularly in poor light, the speed of CMOS camera is slower, but its price very low, and CMOS has power consumption only when the circuit is connected, it is generally used low-end cameras, digital cameras and toys. There is a $20 \mathrm{P}$ plug with $2 \mathrm{~mm}$ pitch in ARM used as extension to connect the camera. The DM9000 is a fully integrated and cost-effective single chip Fast Ethernet MAC controller with a general processor interface, a 10/100M PHY and 4K Dword SRAM.The DM9000 supports 8-bit, 16-bit and 32-bit microprocessor interfaces to internal memory accesses for different processors. The DM9000 also supports IEEE 802.3x full- duplex flow control. A web page has been designed to see the live streaming video.

As mentioned earlier, the client is the subscribed user or the host who needs video monitoring and the SMS alert. The Real-time monitoring is done using a mobile phone or a pc with internet connection. This hardware implementation is similar to the embedded remote surveillance system (Haipeng Chen at el, 2011).

\section{SOFTWARE IMPLEMENTATION OF THE SYSTEM}

The over software design of the system consists of three parts. They are the programming of the micro-controller $1 \& 2$, the Face-recognition module and the 32bit Embedded processor. 


\subsection{Microcontroller $1 \& 2$ control program}

These two micro-controllers are responsible for the system initialization, interrupt handling and the hardware control. The software implementation of the in these micro-controllers is shown in Figure 3 and Figure 4.

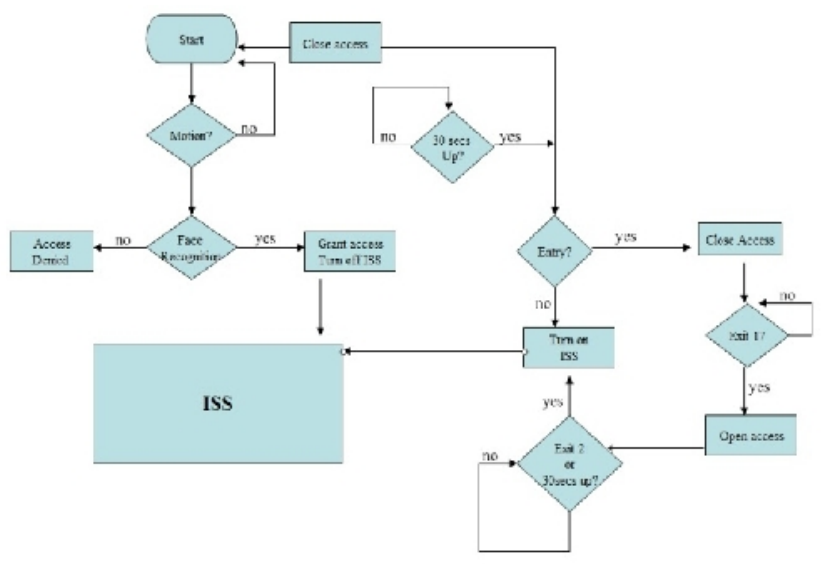

Figure 3 Software Implementation of the System

ISS=Internal Subsystem

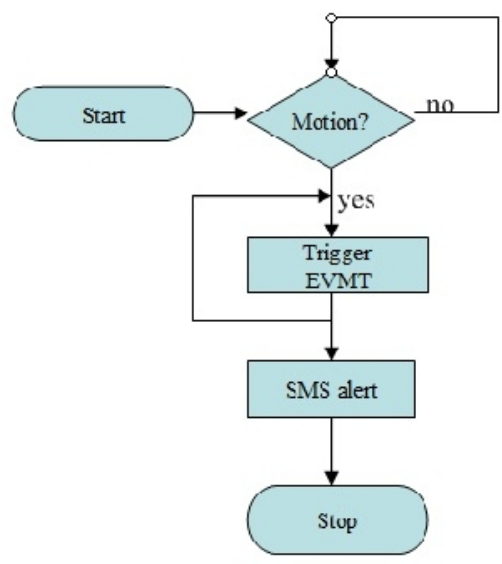

Figure 4 Software Implementation of ISS

The working of the ISS is explained in the latter half of the section.When the system is started, the external sensor keeps sensing motion outside the door and the ISS is turned on as well. If the external sensor detects motion, it triggers the face-recognition module, which in turn makes the camera take a picture of the person in front of the door. The face-recognition module replies the micro-controller with a binary result whether the person is recognized or not. If he/she is recognized, then the access is granted else simply the access is denied, and the ESS after a few seconds, it resets itself. If the access is granted, then the ISS is turned off and the access door is opened for 30 seconds. The timing can be adjusted depending on the need. The person should enter the room and press the entry push button to notify the system that the person has entered the room and if he/she fails to do that, then the access door will be closed and the ISS will be turned On which would lead to the false alarm. When the person wants to leave the room, he/she should 
press the exit 1 push button inside the door for the door to open and on exiting the room he/she should press the exit 2 push button outside the room to notify the system that the person has left the room or the system will automatically close the door in 30 seconds which is also changeable and the ISS will be turned On.

Working of ISS: The ISS has a motion sensor (Internal sensor) which keeps scanning the room for any change in motion. If it detects any motion, it triggers an interrupt. An ISR is executed where the client/user is alerted about the intruder using SMS and triggers the ENVMT.

\subsection{ENVMT}

The embedded network video monitoring terminal is used for video surveillance. This module provides real time video monitoring with lesser jitter and consumes lesser bandwidth and it is proved in china (Wang Kechaoat el,2011). The embedded WinCE operating system (Pang et al, 2009) is selected in the design. There are two modules in the ENVMT. They are the video capturing module and the video streaming module.

Video capture module: Camera captures video frames and sends them to video compression module. It is shown in the Fig 5

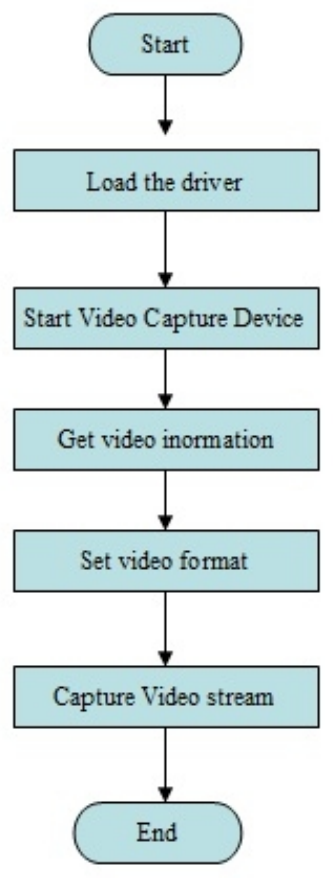

Fig.5 Video capture module of ENVMT

The RGB format of the captured video is converted to $\mathrm{Y} \mathrm{Cb} \mathrm{Cr}$ format for easy processing.Then $\mathrm{Y} \mathrm{Cb} \mathrm{Cr}$ format is compressed using DCT. The floating point values from DCT are rounded off using Huffman coding. It is shown in Fig 6.

Video transmission module: It streams the compressed video through the internet to the client.The block diagram of this module is shown in Fig 7. 
International Journal of Security, Privacy and Trust Management ( IJSPTM) Vol 2, No 5, October 2013

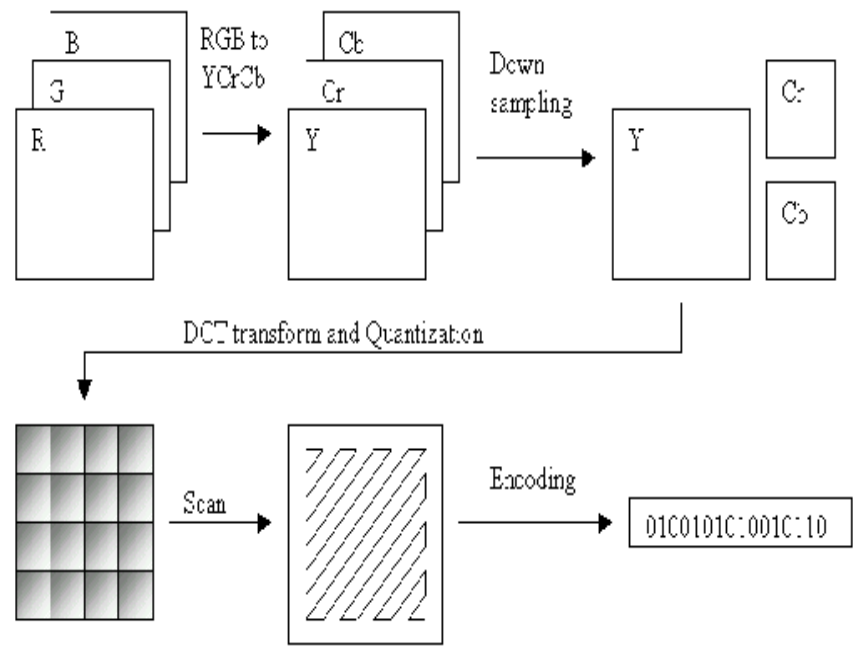

Fig 6 Video compression module of ENVMT

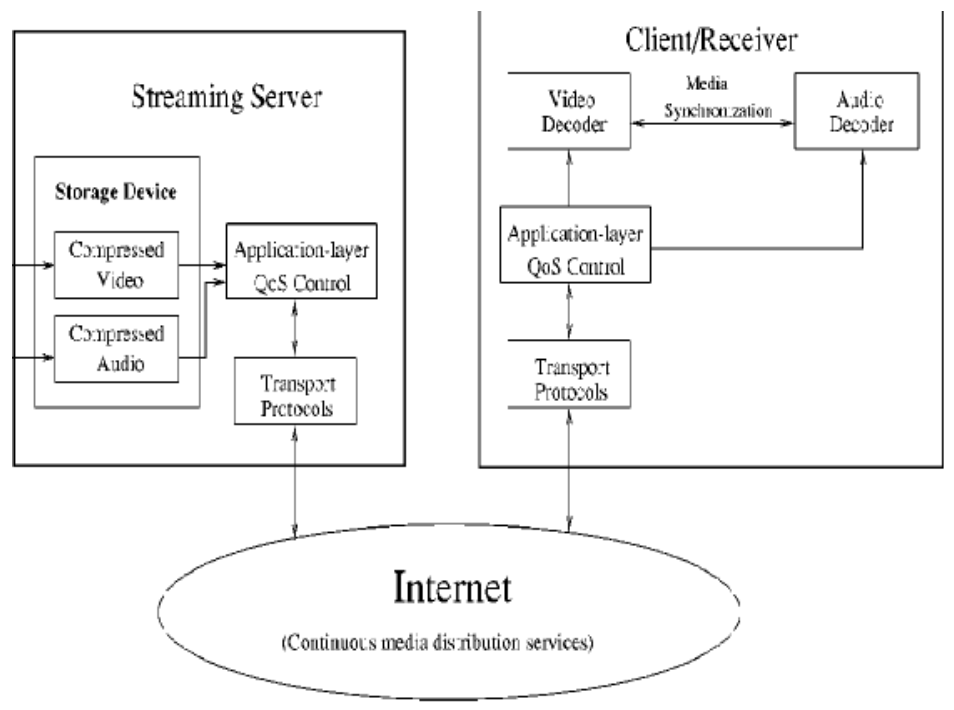

Fig 7 Video transmission module of ENVMT

The compressed video data is stored in a buffer and the application-layer QoS control module adapts the video bit-streams according to the network status and QoS requirements. After the adaptation, the transport protocols packetize the compressed bit-streams and send the videopackets to the Internet. The complete explanation is given in "Streaming video over internet: Approaches and Directions" (Dapeng Wu at el, 2001).

\subsection{Face-Recognition module:}

The Face-recognition module is based on PCA based on Eigen faces and it is programmed using MATLAB/Open CV. It is executed in a PC/FPGA which can be chosen depending on the need or the environment where the system is implemented. The PCA algorithm based on Eigen faces is explained in the figure 6.Principal component analysis transforms a set of data obtained from possibly correlated variables into a set of values of uncorrelated variables called principal 
components. The number of components can be less than or equal to the number of original variables. The first principal component has the highest possible variance, and each of the succeeding components has the highest possible variance under the restriction that it has to be orthogonal to the previous component. We want to find the principal components, in this case eigenvectors of the covariance matrix of facial images. The set of images that are stored in the database are taken as the training set. These set of images are the pictures of the people for whom the access should be granted. From this training set, the mean is calculated and subtracted to get the average vectors from which we can get our covariance matrix and hence the Eigen vectors which are the Eigen faces, E.

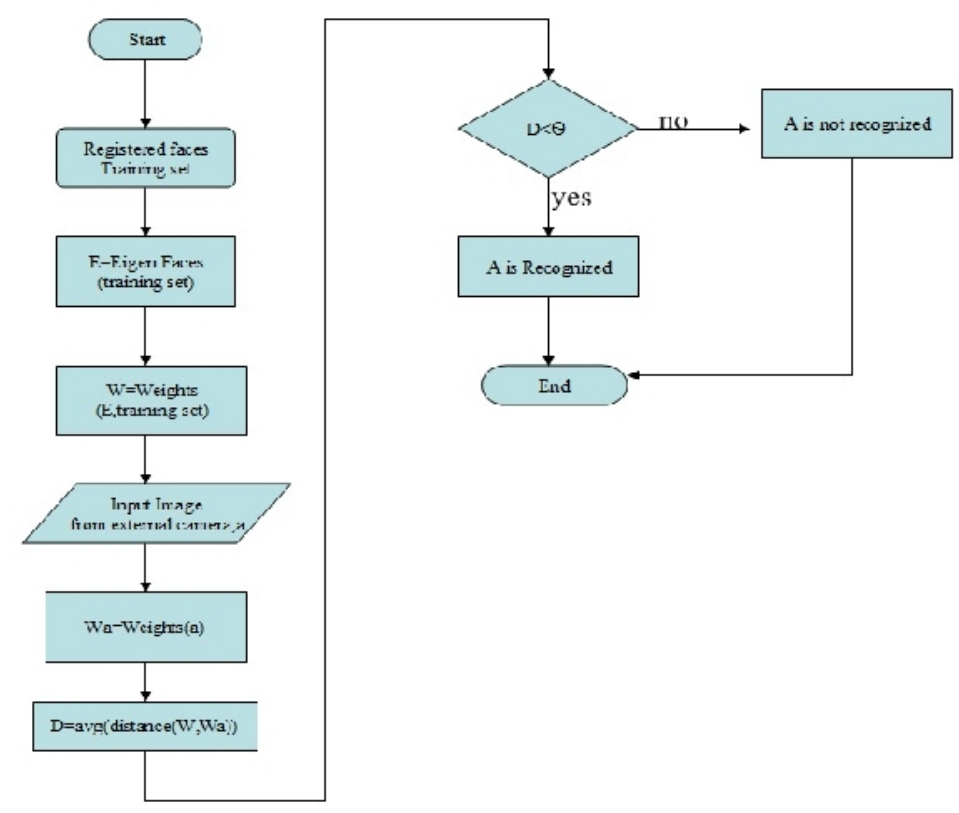

Fig 8. Face recognition using eigen face approach

The weighted matrix (W) is calculated for the training set using the Eigen faces. When an image a is obtained from the external camera, the weighted matrix $\left(\mathrm{W}_{\mathrm{a}}\right)$ for the image a is calculated. Then the weighted matrices are compared to get the distance (D). In this case, the Euclidean distance is calculated. The distance D is compared with the threshold value . If the value is lesser than the threshold value, then the image is recognized, else not recognized. The mathematical formulas for calculating the Eigen vectors, weighted matrix, Euclidean distance is explained in the paper (Marijetaat el, 2012).

\section{SYSTEM TESTING}

"The Network Laboratory, Department of Electronics and Communication Engineering, SRM Easwari Engineering College, Anna University", is taken as the secured room. The ESS results are shown in the Fig 9(a) and (b). 


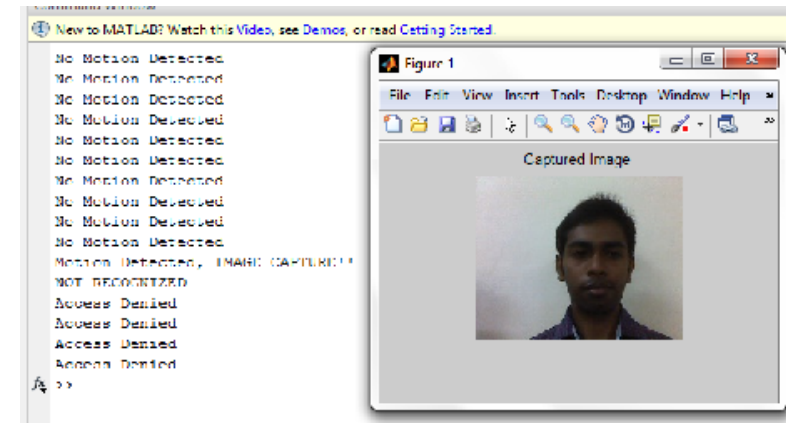

(a)

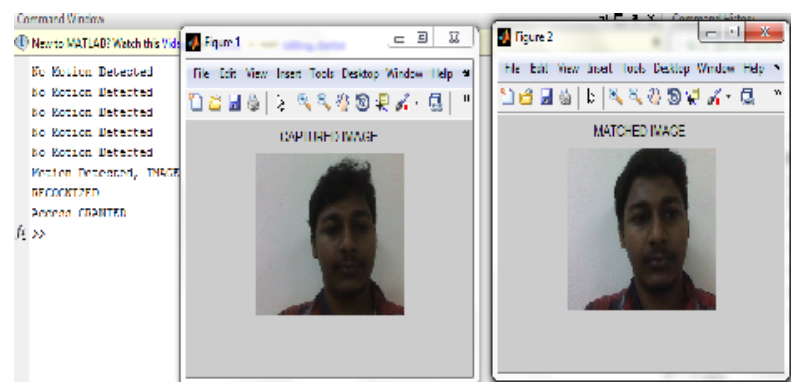

(b)

Fig. 9 ESS Results: (a) Access denied since person not recognized (b) Access granted to authorized personal.

The External sensor scans for movement around the access door. And on detecting motion, it triggers the face-recognition module to capture the image and gives the processed result (Fig 9 (a) and (b)). If access is granted, the ISS is turned Off and then the person may enter the room and press the entry push 1 button and on leaving press the exit push button 1 and after exiting press the exit push button 2, or the system overrides after 30 seconds. The ISS results are shown in the Fig 10 (a), (b) and (c)

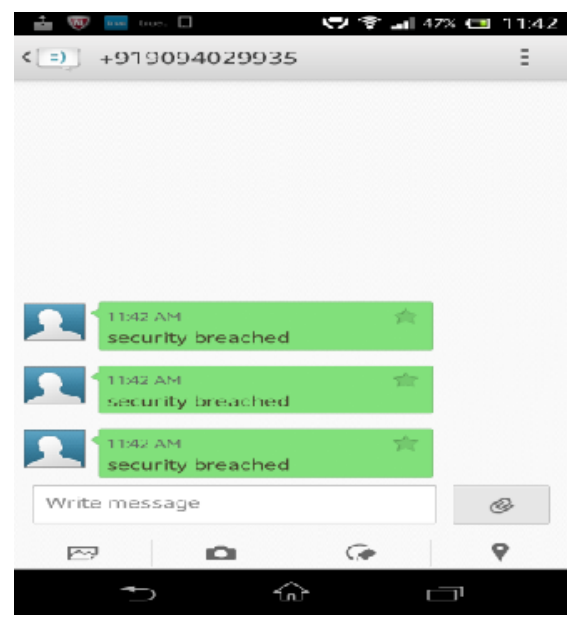

(a) 


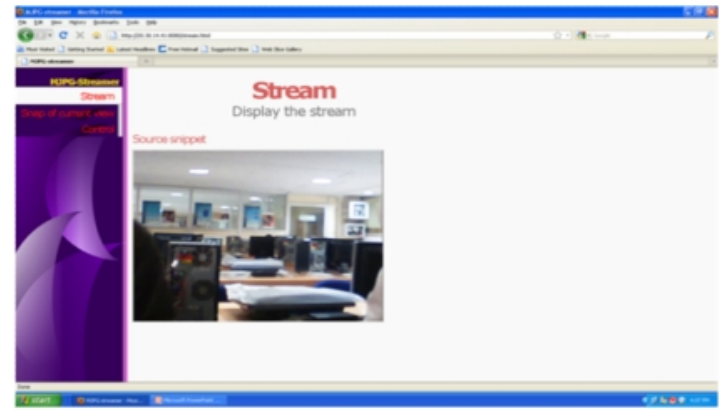

(b)

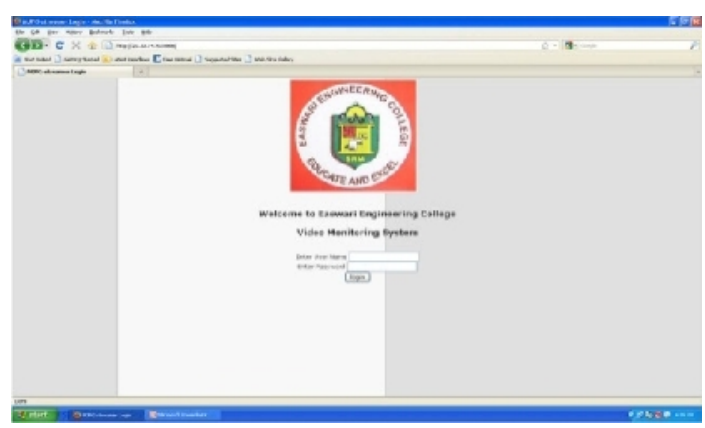

(c)

The ISS detects motion inside the room, and SMS alert (When ISS Internal sensor Fig 10 (a)) is send to the registered phone numbers (clients) and the client can log in from the client log-in page (Fig 10(b)) and watch the real-time live video streaming from the web page (Fig 10(c)).

From the above results shown, we can see that our system has completed all its main functions such as detecting motion, triggering face recognition module and its processing, granting and denying access, SMS alert and real time video monitoring.

\section{CONCLUSION}

Hence, a security system with Face recognition, SMS alert and Embedded network video monitoring terminal has been built which has two sub systems. The External sub-system which can sense motion near the door, capture image of the person, process the image and can grant or deny access to pass through the door (Face Recognition). The Internal sub-system which can sense motion inside the room, send SMS alert and trigger the ENVMT. The ENVMT is implemented using ARM processor, embedded WinCE operating system was selected; the video capture device is made up of S3C2440 processor and CMOS camera (OV9650) with 1.3 million pixel.

Our system has the following advantages: (1) System is easily updateable since all the modules work individually. If there is better face-recognition Our system has the following advantages: (1) System is easily updateable since all the modules work individually. If there is better facerecognition algorithm for the given environment is available, it can be easily updated to the system rather than reprogramming the entire system; (2) only the microcontrollers $1 \& 2$ of the entire system are powered all the time which consumes less voltage and current compared to systems which has its entire module powered; (3) in ENVMT, use CMOS image sensor to capture 
images, their colour and quality are acceptable, although imaging permeability and colour reproduction are not as $\mathrm{CCD}$, it has advantages at low power consumption, price and integration; (4) by using the ENVMT, the captured video is streamed through the internet to the client with much lesser latency and jitter.

Of course, there are still some shortcomings in the system: (1) the entry and exit button system can be automated which can make it more comfortable and user friendly; (2) improved face recognition algorithms can be used which will give better results even with varying environments making the system portable; (3) In addition to face-recognition algorithms, an secondary authorization such as eye lid recognition or finger print recognition can be implemented to make the areas even more secure (4) rather than just using simple PIR sensors, other motion detection modules can be included to make the place more secure (5) By adding temperature sensors, gas sensors, and with a lot more interactive authorization algorithms at the entrance, the system can be changed into an complete home security system rather than just for highly authorized areas.

\section{REFERENCES}

[1] Wang Kechao, Wang Ziangmin, Wang Zhifei, JiaZongfu, Yu Jingwei "Design and implementation of Embedded Network Video Monitoring Terminal” IEEE 2011.

[2] Sutor, S., Matusek, F. , Kruse, F. , Kraus, K. and Reda, R. (2008), 'Large- scale video surveillance system performance parameters and metrics', Internet Monitoring and Protection, ICIMP '08, On $\operatorname{Pagc(s)} 23$ - 30.

[3] Bing Li and Jianping Sun (2009) 'Network Video Monitoring Based on Embedded Linux and VC++', International Conference on Advanced Computer Theory and Engineerings.

[4] Dapeng Wu, Yiwei Thomas Hou,Wenwu Zhu, Ya-Qin Zhang, and Jon M. Peha "Streaming video over Internet: Approaches and Directions" IEEE transactions on circuits and systems for video technology, vol. 11, no. 3, March 2001.

[5] Wang Kechao, Ren Xiangmin, Wang Zhifei, Jia Zongfu and Yu Jingwei, (2011) 'Design and implementation of embedded network video monitoring terminal', Computer Science and Automation Engineering (CSAE), Volume 3, On Page(s) 211-214.

[6] Yakun Liu and Xiaodong Cheng (2010) 'Design and implementation of embedded Web server based on ARM and Linux', Industrial Mechatronics and Automation (ICIMA) Volume 2, On Page(s) 316319.

[7] Yan Liu, Ren FaLi, Cheng Xu and Fei Yu (2008) 'Design and Implementation of Embedded Multimedia Surveillance System',Knowledge Discovery and Data Mining Page(s) 570 - 573

[8] Zhang Songwei and cui ziao (2011) 'Design and implementation of network camera based on TMS320DM365', Artificial Intelligence, Management Science and Electronic Commerce (AIMSEC), Page(s) 3864 - 3867.

[9] Marijeta Slavković1, Dubravka Jevtić1 'Face Recognition Using Eigenface Approach' Serbian Journal Of Electrical Engineering Vol. 9, No. 1, February 2012, 121-130.

[10] M. Turk, A. Pentland: Face Recognition using Eigenfaces, Conference on Computer Vision and Pattern Recognition, 3 - 6 June 1991, Maui, HI , USA, pp. 586 - 591. 